\title{
Aulas híbridas: Escenarios para transformación educativa dentro de la nueva normalidad
}

\section{Hybrid classrooms: Environments for educational transformation in the new normality}

INFORMACIÓN DEL

\section{ARTÍCULO}

Fecha de recepción: 2 de Marzo de 2021.

Fecha de aceptación: 7 de Junio de 2021.

${ }^{1}$ Doctor en Ciencias de la Educación, Universidad Fermín Toro. Docente investigador, Instituto Universitario Pedagógico "Monseñor Rafael Arias Blanco"- Venezuela.

E-mail: arbqto@gmail.com

Código ORCID:

https://orcid.org/0000-0002-0059-7797

CITACIÓN: Prince Torres, A.C. (2021). Aulas híbridas: Escenarios para transformación educativa dentro de la nueva normalidad. Podium, 39, $103-120$.

doi:10.31095/podium.2021.39.7

\section{ENLACE DOI:}

http://dx.doi.org/10.31095/podium.202 1.39 .7

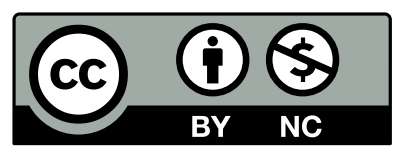

\section{Resumen}

El propósito general de este artículo fue comprender el uso de las aulas híbridas como escenarios para la transformación educativa en el contexto de la nueva normalidad. Para ello, se estructuró una investigación con enfoque cualitativo y diseño documental, complementado con el método fenomenológico hermenéutico, con la finalidad de analizar la información suministrada por UNICEF, UNESCO, y otros autores en sitios web, libros y revistas científicas. Así, se concluyó que la educación híbrida se habría de desarrollar en un contexto que no puede asimilarse a la normalidad que conocieron las personas del planeta Tierra antes de la diseminación del SARS-CoV-2, sino que constituye una adaptación de prácticas previamente realizadas para que los estudiantes puedan de forma presencial o a distancia, seguir con el aprendizaje sin que se comprometa por la imposibilidad de apersonarse en las instituciones.

\section{Palabras Clave:}

Aulas híbridas; transformación; educación; pandemia; nueva normalidad, innovación.

Clasificación JEL: I21, O33.

\begin{abstract}
This work's general purpose was to produce an article that will permit understanding the use of hybrid classrooms as an environment for educational transformation in the context of the new normality. For this purpose, the investigation that was structured followed a qualitative approach with documentary design; this was complemented by the phenomenological and hermeneutical method to analyze information provided by UNICEF, UNESCO and other authors on websites, books and scientific journals. No less than $50 \%$ of the information had five years of antiquity compared to the date when this text was presented. It was concluded that hybrid education would be developed in a context that cannot be assimilated to the normality that people knew before the spread of SARS-CoV-2. It rather constitutes an adaptation of practices previously carried out; in this manner, students can, personally or on-line, continue with their learning process without problems derived from the impossibility of registering in educational institutions.
\end{abstract}

Keywords:

Hybrid classrooms; transformation; education; pandemia; new normality, innovation.

JEL Classification: I21, O33.

$$
103
$$




\section{Introducción}

El año 2020 constituyó uno de los espacios temporales dentro de los cuales se generó mayor incertidumbre a nivel mundial, pues se arrastraron las consecuencias de la proliferación en 2019 del virus SARS-CoV-2 sobre distintas latitudes del planeta, las cuales han sido no sólo individuales o colectivamente físicas, sino que como acota Lozano Vargas (2020) también han sido psicológicas, e incluso sociales, económicas o políticas. De hecho, el fenómeno llegó a establecerse como una pandemia, pues como indica la Organización Panamericana de la Salud -OPS (2020) se la consideró así debido al inminente peligro que representó este acaecimiento sobre la salud pública. Este hecho ha entrañado derivaciones de las cuales como señalan Pizan-Campos Barros-Sevillano, y Yupari-Azabache (2020) el ámbito educacional no ha escapado, porque su faceta presencial se ha visto afectada en cuanto a la necesidad que se ha tenido de aplicar la tecnología para promover el distanciamiento social, lo cual ha generado brechas con respecto a aquellas personas que no pueden acceder correctamente a ella. Sin embargo, el aprendizaje semipresencial o a distancia, parece haber sido la alternativa generalmente aceptada para asegurar la continuidad de las actividades académicas.

Todo esto ocurrió, porque como apunta Covarrubias (2021) el cierre de entes para la educación pública y privada se produjo en más de 190 países para restringir los contagios, por lo que, conforme a cómputos de las Naciones Unidas para la Educación, la Ciencia y la Cultura (UNESCO, citado por Covarrubias, 2021), aproximadamente 1.200 millones de personas dejaron la presencia física en aulas para esperar alternativas que suplieran la necesidad de continuar adelante sin riesgos corporales. Por ello, tiene que acotarse que asegurar el derecho a la educación es una de las metas que deben trazarse los Estados, cuanto más porque es una directriz jurídica, así como política y por ende, aplicar modalidades innovadoras para no comprometerlo es un deber.

De hecho, la Declaración Universal de los Derechos Humanos de la Organización de Naciones Unidas (ONU, 1948), propugna en su artículo 26 que todos tienen derecho a la educación, sobre todo porque debería perseguir el desarrollo de la personalidad en los individuos. Igualmente el Pacto Internacional de Derechos Económicos, Sociales y Culturales de la ONU (1966), estima en su artículo 13 que los Estados Partes de dicho instrumento reconocen que la humanidad debe ser educada y por ello se deben implementar directrices para garantizar incluso la obligatoriedad de ese principio.

Ahora bien, los preceptos nombrados son meramente ilustrativos de una cuestión ampliamente desarrollada en muchos más textos normativos tanto nacionales como internacionales, pero se hace alusión a todo esto para hacer ver que la gobernanza en general debe sistematizar estrategias para consolidar el cumplimiento de sus deberes en cuanto a 
la construcción de saberes en los sujetos. Dicho aspecto es especialmente determinante en un momento dentro del cual se vislumbran cambios globales en vista que, tal como acota Telesur (2020), ya en diciembre del año 2020 se había iniciado con la vacunación en diversos países, incluso de Latinoamérica, lo cual abre una puerta a lo que se denomina como nueva normalidad.

Hernández, López, y López (2020), sostienen que la nueva normalidad se refiere a un concepto dentro del cual se comprende a una situación que no se asemeja a otras conocidas, y que consiste en volver a una normalidad diferente a la asimilada por las personas. Es por ello que en esta etapa pareciera ser necesaria la apertura a los cambios que se suscitarán a futuro y entre esas modificaciones se incluye a la educación, la cual debe adaptarse a las circunstancias. Una de las formas que se ha considerado para seguir con la enseñanza, es por medio de las aulas híbridas, que como expone Gracia (2020) conllevan "...un nuevo modelo híbrido que combine la enseñanza online $\mathrm{y}$ presencial como posible solución" (p. 1), y de esta manera se presenta la mixtura entre el apersonamiento a las aulas junto con el uso de las tecnologías para la comunicación e información, con el propósito de mediar aprendizajes en pandemia.

Es por todo lo esbozado que cabría preguntarse: ¿representa la nueva normalidad una oportunidad para trasformar el fenómeno educacional? Y ¿en qué consiste el uso de las aulas híbridas a todo este respecto?, con lo cual, pueden determinarse los objetivos de este trabajo. Por ello, como propósito general, aquí se planteó comprender el uso de las aulas híbridas como herramientas para el cambio educativo en el contexto de la nueva normalidad.

En todo este sentido, los propósitos específicos de esta investigación son: 1 . Describir las implicaciones de la nueva normalidad; 2. Explicar la definición del cambio educativo y 3 . Ilustrar la esencia de las aulas híbridas.

\section{Revisión de literatura}

\section{Sobre la nueva normalidad}

Cuando se hace referencia a la nueva normalidad como una oportunidad para reconfigurar escenarios, es natural tomar en cuenta el criterio de UNESCO (2020) cuando expone que "...hemos aceptado lo inaceptable durante demasiado tiempo. Nuestra realidad anterior ya no puede ser aceptada como normal. Ahora es el momento de cambiar" (p. 1). En otras palabras, esta etapa de desenvolvimiento social no tendrá las mismas características del ritmo que anteriormente se llevaba de forma comunitaria, por lo que es menester adaptarse a esta novedosa situación.

En virtud de la consideración anterior, Lew y Herrera (2020) exponen que retornar al estado pre-pandemia resulta una configuración de difícil alcance en función de que el daño ocasionado directa e indirectamente por el COVID-19, ha abarcado desde la 
economía hasta incluso, el estado socioambiental. Por ello, “...se sostiene que es necesario construir una 'nueva normalidad', diferente a la 'normalidad pre-pandemia' por ser esta inviable" (Lew y Herrera, 2020, p. 144), lo cual se encuentra alineado con la posición de UNESCO.

Todo esto se sostiene sobre la base fundamental de recordar que la normalidad se define como la "cualidad o condición de normal" (Real Academia Española, 2014, definición 1), es decir, que se refiere a todo aquello que se inserta dentro de lo habitual, pero esta es, tal vez, una visión muy simplista del asunto. Por esto conviene hacer referencia a Matus y Haye (2015), quienes yendo más allá de una reduccionista visión de lo que debería considerarse normal, explican que:

Lo diferente no es un hecho natural ni una construcción aislada, sino que es una producción que se realiza en función de una normalidad imaginada y sostenida por instituciones, discursos y prácticas específicas que se pueden rastrear para comprender sus efectos $\mathrm{y}$ operaciones. La normalidad y la diferencia tienen por tanto un elemento eminentemente político, al igual que los estudios que parten de dichos discursos y prácticas para generar conocimiento sobre lo normal y lo diferente (p. 135).

En otras palabras, la normalidad muchas veces está planteada como un estadio generado por conjunción de elementos que son dictados de acuerdo con las normas o las usanzas que se producen en situaciones, así como estructuras sociales y políticas determinadas. Con ello podría decirse que esa nueva normalidad que se asume condicionará al mundo, podría representar un comienzo de nuevas prácticas que si son reiteradas, calarían en el modus vivendi de las personas conforme se produce el transcurrir del tiempo. Esto es fundamental, porque como señala Lorenzo (2020) desde un punto de vista científico, regresar al estilo de vida conocido antes de la pandemia, podría tardar al menos unos dos años desde que se consolidó su caracterización, y por ello es que se ha producido la transformación digital como medio para ejecutar actividades que antes eran netamente presenciales.

López y Yañez (2020) apuntan que debido a la forzosa salida de los confinamientos que se han convertido en insostenibles, es que se establece el regreso a una relativa normalidad en el desempeño de las actividades, pero eso implica también un proceso de adaptación de rutinas antiguas para continuar hacia adelante. No obstante, para materializarlo, es necesario, de acuerdo con dichos autores, tomar ciertas medidas:

1. Mantener el distanciamiento personal, o lo que es lo mismo, la sana distancia con una aproximación de 1.5 metros entre cada sujeto (Instituto Mexicano del Seguro Social, IMSS, 2020), el uso de mascarillas y el lavado de las manos con agua y jabón por al menos veinte segundos (Fondo de las 
Naciones Unidas para la Infancia, UNICEF, 2020b).

2. Implementar la higiene en el hogar, retirando del cuerpo la ropa y los zapatos al llegar a casa y limpiar todo lo que ingrese en ella, así como sus superficies (UNICEF, 2020a).

3. Evitar el contacto directo (besos, abrazos, etc.) en las relaciones sociales. Según Tala y Vásquez (2020), esto contempla limitaciones en el trato físico, grupal y de desplazamiento.

4. Cuestionar lo que se oye, pero esto principalmente se refiere a descartar rumores e información que no sea oficial sobre el coronavirus y el confinamiento. Según Arroyo-Hernández, QuijanoEscate, y Clavo (2020), esto es crucial especialmente en las redes sociales, por lo que hay que mantener vigilancia sobre ellas.

5. Obtener insumos de publicaciones científicas, que conforme a Rosales (2007), enriquecen a la academia y las ciencias. Con ello se puede descartar también la información falsa sobre el desarrollo del virus.

6. Mantener el cuidado de instituciones de salud y de los trabajadores, pues representan un grupo de riesgo en pandemia debido a su exposición al virus $y$ el contacto constante con personas (Guanche, 2020).

7. Proceder con la vacunación y replicar esta práctica informando a otros sobre ella, lo cual puede lograrse mediante esquemas de cooperación nacionales e internacionales (George, Torres, y O'Ryan, 2020).

8. Acatar las medidas y recomendaciones emanadas desde la gobernanza, pues sus políticas de forma general son tendentes a la reducción de contagios y promoción del distanciamiento social, tal como ocurrió con lo previsto por China (Zhao, 2020).

9. Resguardar el medioambiente para evitar que se generen otras amenazas aunadas al COVID-19, pues incluso inicialmente, esta problemática se produjo por el rompimiento del equilibrio natural que representa una barrera entre la humanidad y algunos patógenos (Gómez, 2020).

Entonces, puede afirmarse que la nueva normalidad no descarta la aplicación de directrices para mantener el cuidado de la humanidad, por lo que no constituiría el regreso al ritmo de vida pre-pandemia. Sin embargo, puede representar la canalización de nuevas prácticas que ayuden a hombres, mujeres y niños a desempeñar su cotidiano accionar.

\section{El cambio educativo}

El cambio debe observarse como un fenómeno que puede abrir puertas sin necesidad de que se trastorne la tranquilidad de las personas. De hecho, Pérez-Vallejo, Vilariño-Corella, y Ronda-Pupo (2017) indican que "el cambio ha existido siempre como una necesidad personal, social y grupal, por 
eso las organizaciones como sistemas abiertos y complejos, deben estar en permanente adaptación al cambio con una elevada capacidad de respuesta" (p. 324) y no sólo las organizaciones deberían asirse a tal cuestión, sino que sobre ello deberían también sostenerse incluso los Estados. No en vano, el cambiar es definido en el Diccionario Ilustrado Océano de la Lengua Española (1994) como "tomar o hacer tomar, en vez de lo que se tiene, algo que lo sustituya" (p. 169), por lo que representa una mutación en lo que habitualmente se acostumbra.

En el ámbito de la educación, también puede presentarse el escenario previamente señalado, y por ello es que en ocasiones se hace un llamado al cambio educativo. Este concepto según Castillo et al (2014), se refiere a la innovación en las prácticas docentes, adaptación de nuevas tecnologías, innovaciones, modificaciones de diseños curriculares, acciones sobre la enseñanza $\mathrm{y}$ otros, que pueden proveer a los educandos aprendizajes y mallas de estudio que resulten más pertinentes para los fines que se proponga el sistema académico.

Hay atmósferas bajo las cuales el cambio educativo es viable para la consecución de los fines del Estado y la sociedad a través de las pautas curriculares, y por ello es que en esas condiciones hay que observarlo como un modo de potenciar lo ya existente, contrariamente a tomarlo como un factor de disrupción de lo ya conocido. Delgado (2010) manifiesta que es "un concepto que implica dos elementos psicoafectivos inherentes al mismo: esperanza $y$ frustración. Un cambio educativo va más allá del deseo o la voluntad, implica planeación, metodología, pero, a pesar de ello, sus posibilidades siempre son inciertas" (p. 1147), con lo cual se sostiene entonces que el proceso de movilización de un sistema a otro puede no ser perfecto, pero tiene potencial para mejorarse siempre y cuando se tomen en cuenta no solo los elementos externos, sino también las manifestaciones de los fueros internos en los implicados.

Es importante, además, indicar que según Ferrer (2008) existe diferencia entre reforma educativa y cambio educativo, puesto que mientras la primera persigue modificar de manera radical la base educacional, el segundo incide sobre las prácticas. De hecho, para el autor existen distintas clases de cambio y entre esas se encuentran:

a) De transformaciones estructurales: Impactan la segmentación, continuidad y perduración de los componentes educativos. Pasan incluso por transformaciones en el conocimiento (Duarte, 2003).

b) De transformaciones curriculares: Tratan la definición, el bosquejo y la ejecución curricular. Chuquilin y Zagaceta (2017) aclaran que las reformas traen consigo cambios curriculares pero que en ocasiones dichos cambios no ocurren en su totalidad porque las reformas que resultan de intereses políticos de los gobiernos en los Estados, a veces no son compatibles con los 
intereses estudiantiles o del profesorado.

c) Organizativos: Son los que inciden sobre las circunstancias bajo las cuales se desarrolla el proceso de aprendizaje en los centros destinados a tal fin. Bolívar (2012), explica que los cambios organizativos deben gestarse para adecuar el cumplimiento del rol institucional en la enseñanza de acuerdo con las necesidades del entorno, especialmente para asegurar el derecho a la educación.

En el siglo XXI y especialmente a raíz de la pandemia, la tecnología (que muchas veces no se encuentra al alcance de todos), se ha convertido en aliada para continuar con las actividades de aprendizaje y por ello, no deben descartarse los cambios educativos desde este punto de vista como factor para la progresión hacia una vida relativamente normal. Por esta razón, resulta lógico hacer un llamado a la tesis de Garcés, Garcés Suárez, y Alcívar (2016), quienes relacionan la integración de las tecnologías de la comunicación e información (TIC) al sistema educativo y el avance ante los retos en una sociedad globalizada, sobre todo por su posibilidad de trasformar los roles de los docentes, educandos y las metodologías aplicadas. Las ventajas de la incorporación tecnológica son, por tanto, las siguientes:

a) Se permite atender a diversidad de estudiantes desde diferentes ámbitos temporales y espaciales: el hogar, la escuela, en horario flexible y con acceso a recursos en red. Estos últimos puntos, de acuerdo con Falcón (2013), son importantísimos para proveer ayuda a aquellas personas que no pueden adecuarse a un esquema rígido de aprendizaje.

b) El impulso del aprendizaje abierto, lo cual se deriva también de la ventaja anterior, y que según Alcibar, Monroy, y Jiménez (2018) también resulta en una forma de proveer conocimientos de forma continua.

c) Complementa las maneras de educar, porque da la posibilidad de llegar a poblaciones remotas con escasa oferta académica, determinando de esta manera un beneficio que puede incidir sobre su futuro inmediato en cuanto a posibilidades intelectuales, laborales, de elevación de nivel de vida, entre otras. Esto es, porque incluso como expone Garduño (2006), la tecnología permite entablar diálogos a distancia, tanto individuales como colectivos.

Una vez esbozado lo que antecede, es cuando entra en ecuación la figura del aula híbrida. En la relación del cambio educativo y el uso de las tecnologías en pandemia y en vía al logro de una nueva normalidad, este modelo debe ser estudiado para determinar su utilidad a todo este respecto.

\section{Relación de las aulas híbridas}

Un aula, desde una perspectiva simplista, se define como una "sala destinada a la enseñanza" (Diccionario Hispánico Universal, 1972, p. 173). Sin embargo, al ir más allá de esta visión, se podría asumir como un espacio dentro del cual se realiza la construcción del conocimiento e igualmente se produce 
una interacción humana desde distintos puntos: académicos, personales, comunicativos, entre otros. A raíz de la crisis producida por el coronavirus, se planteó la implementación de la educación híbrida como una alternativa para la progresión de esta cuestión.

De acuerdo con el diario La República (2020), esta modalidad educativa se circunscribe a lo siguiente:

Actualmente se concibe a la educación híbrida más que el mero resultado de la mezcla de métodos de enseñanza y de formas de gestión presencial y virtual. Más allá de esta simbiosis, se lo visualiza como un modelo propio pedagógico y no fragmentado entre componentes presenciales y virtuales y como el más idóneo en el contexto de la actual disrupción digital para alcanzar con mayor cobertura y calidad (p. 1).

Entonces puede decirse que ese tipo de educación, es producto de la sinergia entre la mediación del aprendizaje a distancia y el que se produce de manera presencial. Una de las estrategias para la consolidación de esta variante educacional es el uso de las aulas híbridas, lo cual de acuerdo con Hernández (2021), permitiría a los alumnos construir sus conocimientos en vivo y también de manera digital, promoviendo del mismo modo los procesos de autonomía estudiantil y la cooperación con el docente para el logro de los propósitos planteados y el alcance de las competencias previstas.

Las aulas híbridas, tal como señala la
Pontificia Universidad Católica Argentina (2020), constituyen canales para "brindar en simultáneo clases virtuales y presenciales en el marco de la pandemia de Covid-19" (p. 1). Esto representa una innovadora forma de hacer frente al confinamiento, en el sentido de que con la búsqueda de una nueva normalidad, se pretendería que no se establezca de manera extremadamente rígida con el fin de que progresivamente se retorne al desempeño de las actividades de la manera que resulte más cercana a la etapa antes de la configuración en la crisis sanitaria.

La Facultad de Informática de Barcelona de la Universidad Politécnica de Cataluña (2020) da cuenta de que, para el desarrollo de este tipo de figura, es necesaria la inversión tecnológica, pues requiere de equipos como cámaras y micrófonos adecuados para el éxito de los objetivos planteados. Estos se adhieren primordialmente a permitir que los profesores hagan acto de presencia dentro de sus aulas físicas institucionales, al tiempo que pueden proveer conocimientos a los estudiantes que por causa de la pandemia no puedan apersonarse dentro de los centros educativos y se encuentren en áreas remotas. No obstante, esta meta pudiera verse condicionada por los recursos que se enfrentan a distintos desafíos de acuerdo con la variedad territorial:

1. De acuerdo con García (2021), el primero de ellos es impulsar la motivación de los estudiantes que, según su criterio, se encuentran agotados luego de pasar por aprendizajes 
preponderantemente en línea, así que se requiere de un nuevo vuelco en la educación que permita recapturar su atención y plantearles retos que los lleven a ser cada día mejores.

2. Preparar a los docentes para que se adapten a la educación híbrida y las aulas en esta modalidad, de forma que diserten sobre estrategias para colaborar y acoplar los conocimientos provistos de manera presencial, pero dando continuidad al uso de las tecnologías (Bejines, 2021).

3. Mantener y mejorar la infraestructura estatal en cuanto a dotación de los centros con tecnología adecuada, incluyendo la cobertura de internet e incluso, la ayuda para que los estudiantes puedan contar con computadores a los fines del aprendizaje (Hernández, 2021).

4. El establecimiento de protocolos gubernamentales orientados a garantizar la integridad personal (especialmente sanitaria) de todos aquellos actores que se manifiesten físicamente dentro de las aulas. Dichos protocolos deben ser variables para adecuarse a la realidad de cada institución e igualmente de cada tipo de estudiante de acuerdo a diferencia de factores como la edad, desarrollo, entre otros (Arce, 2021).

Como se ve, la consolidación del uso de aulas híbridas representa un cúmulo de oportunidades y retos, pero a fin de cuenta su finalidad habrá de ser el logro de aprendizajes en los estudiantes, siendo que especialmente "permitirán seguir en directo una clase desde otro espacio cuando se supere el aforo del aula asignada" (Universidad Pública de Navarra, 2020, p.1), aunque también cuando se imposibilite el traslado de los alumnos. La viabilidad de esta estrategia no es más que una readaptación de los parámetros generales de acuerdo con los cuales "el alumno debe reequilibrarse modificar adecuadamente sus esquemas o construir otros nuevos, de acuerdo a la naturaleza de los contenidos y al tipo de ayuda pedagógica" (Alfaro, 2000, p. 182). En este caso, esa asistencia en materia de pedagogía vendría de parte del docente y las instituciones, la sociedad e incluso el Estado mismo, con el fin de lograr su acoplamiento a esa nueva normalidad que es una puerta para la mutación de usanzas.

\section{Metodología}

Este trabajo fue realizado de acuerdo con un diseño de investigación documental, que según Brito (2015) es aquel en el que se manifiesta un análisis de "...distintos fenómenos de la realidad obtenidos y registrados por otros investigadores en fuentes documentales" (p. 8) y dicha revisión se complementó siguiendo al enfoque cualitativo que "se sustenta en evidencias que se orientan más hacia la descripción profunda del fenómeno con la finalidad de comprenderlo y explicarlo" (Sánchez, 2019 , p. 104). En cuanto a la inserción del esbozo investigativo de documentos dentro de la variante cualitativa, también conviene indicar que conlleva lo siguiente conforme a la Universidad de Jaén (2020, p.1)): 
Investigación documental cualitativa. . . centra su interés en el presente o pasado cercano. Conocer un fenómeno social y cultural a partir de textos escritos (por ejemplo, sobre el problema de género, se podría estudiar la legislación sanitaria, la prensa, las asociaciones de mujeres, etc...

En este trabajo el fenómeno desglosado es el uso de las aulas híbridas en el entorno de la pandemia, en miras a las perspectivas de su desarrollo al referir a una nueva normalidad para la humanidad. Igualmente se dilucidaron a través del método fenomenológico hermenéutico los textos observados y la realidad expresada en ellos, tal como señalan Tiusabá, Barreto, y Cerón (2019) al explicar su esencia. Además, con referencia al método hermenéutico, Ruedas, Ríos, y Nieves (2009) consideran que es un sistema general de interpretación sobre el espíritu en todas sus variantes, e incluso, que por ello tiene un espectro más amplio que el de la psicología.

También es conveniente indicar que las fuentes documentales utilizadas constaron de cuerpos contenidos en índices y bases de datos como SciELO, sitios web de organismos oficiales, universidades y portales informativos de reconocido prestigio, incluso con insignia de verificación en redes sociales como Twitter, Facebook o Instagram, así como publicaciones físicas en papel. Con respecto a las técnicas operacionales para manipulación de documentos que se ejecutaron, se puede referir a la observación, la lectura en profundidad, el resumen y subrayado. Además, fue conveniente el análisis de los datos recabados a través del análisis crítico, que, de acuerdo con Moreno, Puerta, Cuervo, y Cuéllar (2016) se orienta a comprender las ideas plasmadas en lo leído. Cabe destacar que las fuentes consultadas, entre los criterios para su elección, debían al menos en un 50\% del total de ellas, tener una antigüedad máxima de 5 años con respecto a la fecha de presentación de este artículo, y a nivel cualitativo, tenían que contener información que permitiera comprender el fenómeno de la implementación de las aulas híbridas en el contexto de la pandemia y la nueva normalidad.

De acuerdo con el producto investigativo configurado y conforme a un proceso reflexivo del escritor, se desprendieron ciertas categorías que determinaron el contenido del cuerpo del trabajo separando el tópico de estudio, las implicaciones del tema y la forma de abordar la temática, todo conforme con la finalidad que, desde el punto de vista cualitativo, se persiguió para comprender el fenómeno. Estas categorías fueron las siguientes:

a) Manifestación situacional: Esta categoría se encuentra referida al contexto dentro del cual se estudió el tema. En este caso, se trató el ámbito de la nueva normalidad.

b) Reconducción: Se refiere a la mutación de las prácticas para adaptarlas a los requerimientos sociales. Aquí se trata de un replanteamiento de la educación. 
c) Estrategias innovadoras: Versa sobre los instrumentos considerados con la pandemia, de forma que sean soporte para colaborar con la continuación de las actividades sociales. La estrategia innovadora para este estudio, es la implementación de las aulas híbridas.

Ahora bien, una vez esbozados los aspectos metodológicos anteriores, debe procederse a explicar el sustento para la relación de este artículo. Por ello, en primer lugar, se refiere a la nueva normalidad como punto para el desarrollo de las actividades educativas humanas.

\section{Resultados y discusión}

Conforme a la información recopilada, puede afirmarse que en este trabajo se determinaron los siguientes resultados:

1. Se comprendió que la nueva normalidad no implica la vuelta al mismo estilo de vida antes del surgimiento de la COVID-19, sino que es simplemente una readaptación de las actividades cotidianas a las medidas para resguardar la integridad de las personas mientras se supera esta problemática. Este aspecto, se concatena con el apartado previamente desarrollado sobre dicha nueva normalidad, en el cual se explica tal concepto y su alcance, especialmente conforme con los aportes realizados por autores como Lew y Herrera (2020), López y Yañez (2020) e incluso UNESCO (2020), entre otros.

2. Se interpretó que la pandemia constituyó una forma de implementar la creatividad para seguir adelante con las prácticas de la humanidad, pues especialmente al introducirse el uso de las tecnologías en una estructura tradicionalista, se ha fomentado el cambio educativo que se establece en otro espacio de este artículo, tal como se refirió con las afirmaciones sustentadas por Castillo et al (2014).

3. Igualmente, de acuerdo con las fuentes consultadas se consideró que el cambio educativo pasa principalmente por la modificación de las prácticas para el aprendizaje, con alineación a los objetivos personales, sociales y estatales. Dicha cuestión desarrollada en el fragmento llamado el cambio educativo, fue suficientemente explicada con el soporte de las afirmaciones de Delgado (2010) y Ferrer (2008).

4. Gracias a las fuentes analizadas se definió que la pandemia constituyó una forma de revitalizar al uso de la tecnología para la educación. Esto es fácilmente observable a lo largo de la revisión documental configurada, pues constantemente se expone que la educación híbrida une a la presencialidad con el uso de las TIC.

5. En los documentos desglosados se conceptualizó al aula híbrida como un espacio donde se conjugan el uso de las tecnologías para la comunicación e información y el apersonamiento físico de los actores educativos, de manera que en el contexto de la pandemia, pueda continuarse con la educación tanto de personas que puedan asistir presencialmente a clases, como aquellas que no puedan. Esta 
acotación, se fundamentó en el fragmento dedicado a la relación de las aulas híbridas, en consonancia con las aseveraciones como las de Hernández (2021) o el suplemento informativo del diario La República (2020), con base en las definiciones generales de aula que son conocidas y aceptadas.

6. En los textos estudiados se observó que para el establecimiento de las aulas híbridas se requiere la confluencia de recursos materiales, financieros, intelectuales y humanos que permitan su correcta dotación y manipulación. Testimonios que sirven para sostener esta consideración, se encuentran en documentos publicados y pertenecientes a la Pontificia Universidad Católica Argentina (2020), la Universidad Pública de Navarra (2020) o la Facultad de Informática de Barcelona de la Universidad Politécnica de Cataluña (2020), las cuales como instituciones que han considerado a la modalidad de educación híbrida, cuentan con su ejecución a través de las acciones desarrolladas para llevarla a cabo.

Ahora bien, estas cuestiones dan a entender que, en el inicio y transcurso de una etapa de nueva normalidad, dentro de la cual las personas ya no se encuentren en situación de encierro dentro de sus hogares para protegerse del coronavirus, las aulas híbridas constituyen espacios dentro de los cuales se pueden iniciar nuevamente las relaciones interpersonales que tan necesarias son para los seres humanos. No implica ello un relajamiento de las medidas de bioseguridad, pero representa un paso hacia adelante para que los estudiantes logren competencias más allá que las puramente académicas.

Asimismo, se entiende que el papel de la coordinación entre estudiantes y profesores es necesario para el éxito de la aplicación de las aulas híbridas, de manera que sepan cuales acciones tomar, los horarios para ejecutarlas y sus circunstancias, de modo que todos los que participen presencialmente $\mathrm{o}$ a distancia, tengan las mismas oportunidades para aprender. Debe recordarse aquí que este mecanismo no solo se aplicaría hacia estudiantes que no pudieran asistir a sus clases de forma física, sino también cuando las aulas se colmen de personas, así que incluso la armonización en cuanto a rotación de la presencialidad podría definir el logro de los objetivos.

Igualmente debe recalcarse que, de acuerdo con los autores, la nueva normalidad implica cambios de paradigmas, y de ello no escapa el aspecto educacional, por lo que la planificación para el aprendizaje híbrido debe considerar que se desenvolverá en el entorno de una prolongación de las constantes medidas para frenar al SARS-CoV-2. Además, el rol del Estado jugaría un papel fundamental siempre que pueda proveer ayuda de la siguiente manera:

1. Establecer aportes financieros destinados a potenciar las plataformas tecnológicas dentro de sus territorios.

2. Ayudar con alivios financieros a las instituciones educativas, de manera 
que puedan dotarse adecuadamente para la construcción de las aulas híbridas a través de recursos tecnológicos, habilitación de espacios para estas prácticas y la correcta preparación de los docentes ante este escenario.

3. Indicar claramente las directrices de seguridad que en general se han de seguir como protocolos de actuación para seguir con la educación ante la nueva normalidad.

4. Abrirse a sugerencias de los actores educativos, de manera que se optimicen las aulas híbridas de acuerdo con sus experiencias. Con ello se enriquecerían las líneas de acción en los distintos países.

Para finalizar este segmento, cabría decir que es necesario realizar seguimiento a la implementación de las aulas híbridas. Por ello podría resultaría pertinente realizar futuros estudios científicos cuantitativos y cualitativos que recojan datos sobre la eficacia que ellas han tenido. Con este tipo de información sería posible realizar la reingeniería de todos aquellos aspectos que no resultaran adecuados en un primer momento.

\section{Conclusiones}

Los nuevos comienzos no siempre resultan fáciles y muchas veces pueden encontrarse con obstáculos que impidan la fluidez hacia el asentamiento de nuevas prácticas o circunstancias. Con lo ocurrido a raíz de la pandemia por coronavirus se ha puesto a prueba la capacidad del ser humano para sobrepasar escollos y encontrar formas de sobrevivir ante los problemas que puedan surgir, pero hasta ahora, a pesar de las experiencias negativas, se ha continuado con la vida de una forma relativamente aceptable apartando las secuelas de salud y muerte que se han observado.

La educación no puede detenerse pues es el motor de la sociedad, y por ello es deber de todos los actores educativos realizar esfuerzos mancomunados para dar continuidad a tan loable labor ante las adversidades. En este trabajo se han planteado las implicaciones de realizarlo por medio de la estructuración de las aulas híbridas. Por ello, de acuerdo con los objetivos planteados, se generaron las siguientes conclusiones:

a) La nueva normalidad es una reconfiguración de todo lo que ya se conoce, para adaptarlo a la emergencia sanitaria que continúa en 2021.

b) El cambio educativo en cuanto a las prácticas y estrategias bajo tal figura, ha de permitir la flexibilización de la comunidad educacional para entrar en la etapa de la nueva normalidad.

c) Las aulas híbridas pueden formar parte de ese repensar educativo en la nueva normalidad, puesto que su esencia es la unión del uso en la tecnología con la presencialidad, de manera que se cubran las necesidades de la mayor cantidad de estudiantes posibles.

En la realización de este trabajo, las principales limitaciones que se encontraron para estudiar el modelo de 
educación híbrida, fueron la restricción de información al respecto en el contexto de la pandemia y la nueva normalidad, ya que no existe la misma abundancia de datos bibliográficos sobre tal tópico comparado con otros temas de naturaleza educativa, e igualmente fue limitante el carácter reciente que tiene el auge de esta estrategia, pues contribuyó con lo indicado primeramente en este párrafo, aunque ello no impidió que se sustentara este escrito. Tal vez con el transcurrir del tiempo se solventen las restricciones que conllevan tales factores.

Además, en función de todo lo desarrollado, se estableció un marco de futuras líneas de investigación, relativo a la potencialidad exploratoria que se tiene en cuanto al impacto de las aulas híbridas. En este sentido, en subsiguientes estudios podrían desglosarse la recepción que han tenido las aulas híbridas en el contexto de la nueva normalidad; las implicancias psicológicas sobre los estudiantes, con respecto a la aplicación de este modelo en contraste con los paradigmas que se encontraban asentados en sus fueros internos en el contexto pre-pandemia; el impacto sobre la noción de igualdad en la facilitación de conocimientos entre aquellos educandos que de acuerdo con esta perspectiva pueden participar de manera presencial en contraposición con aquellos que deben hacerlo a distancia; la factibilidad de propuestas para mejorar el desempeño de la educación híbrida, pudiendo presentarse con enfoque hacia las instituciones de aprendizaje o incluso el Estado, entre otros temas que se vinculan con la innovadora estructura aquí tratada.
Ahora bien, como señala Marín (2012), es importante comprender que “...lo más importante es la intensidad con la que vivamos, porque muchos viven una vida superficial, sin amar ni servir..." (p. 160), y en este sentido, la lección que queda para sacar a la luz elementos novedosos dentro de la praxis educativa, es que experimentando la etapa denominada como "nueva normalidad", podrán surgir nuevas formas en las que se podrán modificar esquemas para facilitar la existencia de los seres humanos. Por ello, la apertura hacia nuevas experiencias es necesaria, de forma que la maleabilidad sea la mejor herramienta para escudarse de futuros fenómenos disruptivos de la cotidianidad que pudieran causar perturbaciones en los años por venir.

\section{Referencias}

Alcibar, M., Monroy, A., y Jiménez, M. (2018). Impacto y Aprovechamiento de las Tecnologías de la Información y las Comunicaciones en la Educación Superior. Información tecnológica, 29(5), 101-110. DOI: https://dx.doi.org/10.40 67/S0718-07642018000500101

Alfaro, M. (2000). Evaluación del aprendizaje. Venezuela: FEDUPEL.

Arce, J. (2021). Desafíos para pedagogía innovadora en el contexto de continuidad de la crisis sanitaria. Boletín Opiniones Iberoamericanas en Educación de la Universidad Miguel de Cervantes, (17), 28-29. https://www.linkedin.com/feed/up date/urn:li:activity:67609134194922127 36/

Arroyo-Hernández, H., Quijano-Escate, R., y Clavo, M. (2020). Análisis de las respuestas a rumores sobre COVID-19 en 
Perú. Revista Cubana de Información en Ciencias de la Salud, 31(3), 1579. http://scielo.sld.cu/scielo.php?script=sci arttext\&pid=S2307-21132020000300005 \&lng=es\&tlng=es.

Bejines, C. (2021). Los desafíos docentes para implementar una educación híbrida. Boletín Opiniones Iberoamericanas en Educación de la Universidad Miguel de Cervantes, (17), 30-31. https://www. linkedin.com/feed/update/urn:li:activity: 6760913419492212736/

Bolívar, M. (2012). Por una renovación organizativa de los centros escolares. Revista mexicana de investigación educativa, 17(52), 313-320. http://www.scielo.org. $\mathrm{mx} /$ scielo.php?script $=$ sci_arttext\&pid $=\mathrm{S}$ 1405-66662012000100014 \&lng=es\&tlng $=\mathrm{es}$

Brito, A. (2015). Guía para la elaboración, corrección y asesoramiento de trabajos de investigación. San Tomé: Universidad Nacional Experimental Politécnica de la Fuerza Armada Bolivariana.

Castillo, M., Hawes, G., Castillo, S., Romero, L., Rojas, A., Espinoza, M., y Oyarzo, S. (2014). Cambio educativo en las Facultades de Medicina. Revista médica de Chile, 142(8), 1056-1060. DOI: https://dx.doi.org/10.4067/S0034-988720 14000800013

Chuquilin, J., y Zagaceta, M. (2017). El currículo de la educación básica en tiempos de transformaciones: los casos de México y Perú. Revista mexicana de investigación educativa, 22(72), 109-134. http://www.scielo.org.mx/scielo.php?scri $\mathrm{pt}=$ sci_arttext\&pid=S1405-66662017000 100109 \&lng $=$ es \&tlng $=$ es

Covarrubias, L. (2021). Educación a distancia: transformación de los aprendizajes. Telos: revista de Estudios Interdisciplinarios en Ciencias Sociales, 23(1), 150-160. DOI: www.doi.org/10.36390/telos231.12
Delgado, A. (2010). Cambio educativo. Un tema crucial. Revista mexicana de investigación educativa, 15(47), 1147-1152. http://www.scielo.org.mx/s cielo.php?script $=$ sci_arttext\&pid $=\mathrm{S} 1405$ $6666201000040000 \overline{7} \& \operatorname{lng}=\mathrm{es} \& \operatorname{tlng}=\mathrm{es}$.

Diccionario Hispánico Universal. (1972). Diccionario Hispánico Universal (decimoséptima edición). México: W.M. Jackson Inc. Editores.

Diccionario Ilustrado Océano de la Lengua Española. (1994). Diccionario Ilustrado Océano de la Lengua Española. España: Océano Grupo Editorial.

Duarte, J. (2003). Ambientes de aprendizaje: Una aproximación contextual. Estudios pedagógicos (Valdivia), (29), 97-113. DOI: https://dx.doi.org/10.4067/S071807052003000100007

Facultad de Informática de Barcelona-Universidad Politécnica de Cataluña. (2020). Aulas docentes [nota web]. FIB. Recuperado de https://www.fib.upc.edu/es/la-fib/espacio s/aulas-docentes

Falcón, M. (2013). La educación a distancia y su relación con las nuevas tecnologías de la información y las comunicaciones. MediSur, 11(3), 280-295. http://scielo.sld.cu/scielo.php?script=sci arttext\&pid=S1727-897X201300030000 $6 \& \operatorname{lng}=$ es\&tlng=es.

Ferrer, A. (2008). Evaluación y cambio de los sistemas educativos: la interacción que hace falta. Ensaio: Avaliação e Políticas Públicas em Educação, 16(59), 275-296. DOI: https://dx.doi.org/10.1590/S010440362008000200007

Garcés, E., Garcés Suárez, E., y Alcívar, O. (2016). Las tecnologías de la información en el cambio de la educación superior en el siglo XXI: Reflexiones para la práctica. Revista Universidad y Sociedad, 8(4), 
171-177. http://scielo.sld.cu/scielo.php? script $=$ sci_arttext\&pid=S2218-36202016 $000400023 \& \operatorname{lng}=\mathrm{es} \&$ tlng $=\mathrm{es}$.

García, E. (2021). Desafíos para pedagogía innovadora en el contexto de continuidad de la crisis sanitaria. Boletín Opiniones Iberoamericanas en Educación de la Universidad Miguel de Cervantes, (17), 32-33. https://www.linkedin.com/feed/up date/urn:li:activity:67609134194922127 $36 /$

Garduño, R. (2006). Objetos de aprendizaje en la educación virtual: una aproximación en bibliotecología. Investigación bibliotecológica, 20(41), 161-194. http://www.scielo.org. $\mathrm{mx} /$ scielo.php?script $=$ sci_arttext\&pid $=\mathrm{S}$ 0187-358X2006000200008\&lng=es\&tln $\mathrm{g}=\mathrm{es}$

George, S., Torres, J., y O'Ryan, M. (2020). Pandemia y vacunas, ¿quimera o realidad? Revista médica de Chile, 148(4), 427-428. DOI: https://dx.doi.org/ $10.4067 / \mathrm{s} 0034-98872020000400427$

Gómez, L. (2020). El desafío ambiental: enseñanzas a partir de la COVID-19. MEDISAN, 24(4), 728-743. http://scielo.sld.cu/scielo. php? script $=$ sci_arttext\&pid=S1029-3019 $2020000400728 \& \operatorname{lng}=\mathrm{es} \& \operatorname{tlng}=\mathrm{es}$.

Gracia, M. (2 septiembre, 2020). Aulas híbridas: tecnología y educación en un mundo pandémico [artículo web]. elPeriodico. Recuperado de https://www.elperiodico. com/es/activos/innovadores/20200902/au las-hibridas-wacom-vuelta-cole-educacio n-pandemia-8095324

Guanche, H. (2020). COVID-19. Un reto para los profesionales de la salud. Revista Habanera de Ciencias Médicas, 19(2), e3284. http://scielo.sld.cu/scielo.php? script $=$ sci_arttext\&pid=S1729-519X202 $0000200001 \& \operatorname{lng}=\mathrm{es} \& \operatorname{tlng}=\mathrm{es}$.

Hernández, R. (2021). Educación híbrida: Reinvención de nuestra praxis educativa en momentos de crisis. Boletín Opiniones Iberoamericanas en Educación de la Universidad Miguel de Cervantes, (17), 7-8. https://www.linkedin.com/feed/upda te/urn:li:activity:6760913419492212736/

Hernández, W. (2021). Condiciones sociales y retos administrativos para el establecimiento del modelo híbrido de educación en Colombia. Boletín Opiniones Iberoamericanas en Educación de la Universidad Miguel de Cervantes, (17), 24-25. https://www. linkedin.com/feed/update/urn:li:activity: 6760913419492212736/

Hernández, Y., López, L. y López, E. (2020). Terminología y escritura en tiempos de COVID-19. CorSalud, 12(2), 184-188. http://scielo.sld.cu/scielo.php?script=sci arttext\&pid=S2078-71702020000200184 \&lng=es\&tlng=es.

IMSS (Junio, 2020). Sana distancia y medidas generales de higiene, principales recomendaciones ante el retorno a la Nueva Normalidad [artículo web]. IMSS. Recuperado de http://www.imss.gob.mx/ prensa/archivo/202006/382

La República (Junio 22, 2020). La enseñanza de la pandemia: la educación híbrida. $L a$ República. https://www.republica.com. uy/la-ensenanza-de-la-pandemia-la-educ acion-hibrida-id771648/

Lew, D., y Herrera, F. (2020). Normalidad postpandemia: ¿una nueva normalidad socio-ambiental o adiós a la normalidad? Observador Del Conocimiento, 5(2), 144-167. http://www.oncti.gob.ve/ojs/ index.php/rev_ODC/article/view/53

López, R., y Yañez, G. (2020). La nueva normalidad: elementos indispensables [nota web]. Universidad Veracruzana. Recuperado de https://www.uv.mx/investigacion/general /nota-la-nueva-normalidad/

Lorenzo, M. (09 agosto, 2020). Los científicos 
apuntan que tras la pandemia la normalidad puede tardar dos años como mínimo. elPeriodico. Extraído de https://www.elperiodico.com/es/sociedad /20200809/expertos-valencia-volver-a-la -normalidad-tras-covid19-minimo-dos-a nos- 8069780

Lozano-Vargas, A. (2020). Impacto de la epidemia del Coronavirus (COVID-19) en la salud mental del personal de salud y en la población general de China. Revista de Neuro-Psiquiatría, 83(1), 51-56. DOI: https://dx.doi.org/10.20453/rnp.v83i1.36 87

Marín, N. (2012). Vencer al enemigo perdonándolo. Venezuela: Editorial Ignaka C.A.

Matus, C.,y Haye, A. (2015). Normalidad y diferencia en la escuela: Diseño de un proyecto de investigación social desde el dilema político-epistemológico. Estudios pedagógicos (Valdivia), 41(especial), 135-146. DOI: https://dx.doi.org/10.40 67/S0718-07052015000300009

Moreno, E., Puerta, C., Cuervo, C., y Cuéllar, A. (2016). Análisis crítico de literatura científica. Una experiencia de la Facultad de Ciencias de la Pontificia Universidad Javeriana. Voces y Silencios. Revista Latinoamericana de Educación 7(2), 74-97. DOI: https://doi.org/10.18175/v ys 7.2.2016.06

Organización de Naciones Unidas (1948). Declaración Universal de los Derechos Humanos. UN. Recuperado de https://www.un.org/es/universal-declarati on-human-rights/

Organización de Naciones Unidas (1966). Pacto Internacional de Derechos Económicos, Sociales y Culturales. Naciones Unidas Derechos Humanos, Oficina del Alto Comisionado. Extraído de https://www. ohchr.org/sp/professionalinterest/pages/c escr.aspx
Organización Panamericana de la Salud (2020). La OMS caracteriza a COVID-19 como una pandemia [nota web]. OPS. Recuperado de https://www.paho.org/hq/ index.php?option $=$ com_content\&view $=\mathrm{a}$ rticle\&id=15756: who-characterizes-covi d-19-as-a-pandemic\&Itemid=1926\&lang=es

Pérez-Vallejo, L., Vilariño-Corella, C., y Ronda-Pupo, G. (2017). El cambio organizacional como herramienta para coadyuvar con la implementación de la estrategia. Ingeniería Industrial, 38(3), 323-332. http://scielo.sld.cu/scielo.php?script $=$ sci arttext\&pid=S1815-59362017000300010 $\& \operatorname{lng}=\mathrm{es} \& \operatorname{tng}=\mathrm{es}$

Pizan-Campos, E., Barros-Sevillano, S. y YupariAzabache, I. (2020). Impacto del COVID-19 en la educación de los estudiantes de medicina del Perú. Revista de la Facultad de Medicina Humana, 20(3), 534-535. DOI: https://dx.doi.org/ 10.25176/rfmh.v20i3.2959

Pontificia Universidad Católica Argentina (29 diciembre, 2020). La UCA prepara aulas híbridas para brindar clases virtuales y presenciales en simultáneo [artículo web]. UCA. Recuperado de http://uca.edu.ar/es/noticias/la-uca-prepar a-aulas-hibridas-para-brindar-clases-virtu ales-y-presenciales-en-simultaneo

Real Academia Española. (2014). Normalidad. Diccionario de la Lengua Española. Recuperado de https://dle.rae.es/normalidad

Rosales, L. (2007). El acceso a fuentes de información científica. Universidad, Ciencia y Tecnología, 11(45), 166. http://ve.scielo. org/scielo.php?script $=$ sci_arttext\&pid $=\mathrm{S}$ 1316-48212007000400001\&lng=es\&tlng $=\mathrm{es}$

Ruedas, M., Ríos, M., y Nieves, F. (2009). Hermenéutica: La roca que rompe el espejo. Investigación y Postgrado, 24(2), 181-201. http://ve.scielo.org/scielo.php? script $=$ sci_arttext\&pid=S1316-00872009 
000200009\&lng=es\&tlng=es

Sánchez F. (2019). Fundamentos epistémicos de la investigación cualitativa y cuantitativa: Consensos y disensos. Revista Digital Investigación y Docencia 13(1), 101-122. http://www.scielo.org.pe/scielo.php?scrip $\mathrm{t}=$ sci_arttext\&pid=S2223-251620190001 $0000 \overline{8}$

Tala, Á., y Vásquez, E. (2020). Conexión en tiempos de COVID-19. Revista médica de Chile, 148(4), 557-558. DOI: https://dx.doi.org/ 10.4067/s0034-98872020000400557

Telesur (25 diciembre, 2020). Inicia vacunación anti-Covid en varios países de Latinoamérica [artículo web]. Telesur. Recuperado de https://www.telesurtv. net/news/inicia-vacunacion-anti-covid-va rios-paises-latinoamerica-20201225-003 3.html

Tiusabá, B., Barreto, R., y Cerón, L. (2019). Hermenéutica, realidad y método en la disciplina de las Relaciones Internacionales. Revista mexicana de ciencias políticas y sociales, 64(236), 217-237. DOI: https://doi.org/10. 22201/fcpys.2448492xe.2019.236.63223

UNESCO. (2020). La campaña "La Nueva Normalidad" de la UNESCO [nota web]. UNESCO. Recuperado de https:// es.UNESCO.org/campaign/nextnormal

UNICEF. (2020a). Consejos de limpieza e higiene para mantener el coronavirus (COVID-19) fuera de tu hogar [nota web]. UNICEF. Recuperado de https://www.UNICEF.org/es/coronavirus/ consejos-limpieza-higiene-para-mantener -coronavirus-covid-19-fuera-de-tu-hogar

UNICEF (2020b). Todo lo que debes saber sobre el lavado de manos para protegerte del coronavirus (COVID-19) [nota web]. UNICEF. Recuperado de https://www. UNICEF.org/es/coronavirus/todo-lo-quedebes-saber-sobre-el-lavado-de-manos-p ara-protegerte-del-coronavirus-covid19

Universidad de Jaén. (2020). Diseño documental [página web]. Recuperado de http:// www.ujaen.es/investiga/tics_tfg/dise_doc umental.html

Universidad Pública de Navarra (2 septiembre, 2020). La UPNA se dota de medio centenar de "aulas híbridas" para garantizar la docencia presencial de sus más de 8.000 estudiantes. Universidad Pública de Navarra [artículo web]. Recuperado de http://www.unavarra.es/ sites/actualidad/contents/noticias/2020/0 9/20-09-02/la-upna-se-dota-de-medio-ce ntena.html

Zhao, G. (2020). Tomar medidas preventivas inmediatamente: evidencia de China sobre el COVID-19. Gaceta Sanitaria, 34(3), 217-219. DOI: https://dx.doi.org/ 10.1016/j.gaceta.2020.03.002 